\title{
REVIEW OF CRYSTAL DIFFRACTION AND ITS APPLICATION TO FOCUSING ENERGETIC GAMMA RAYS
}

\author{
Robert K. Smither, Patricia B. Fernandez, Timothy Graber \\ Advanced Photon Source, Argonne National Laboratory, * \\ 9700 S. Cass Ave., Argonne, IL 60439 USA
}

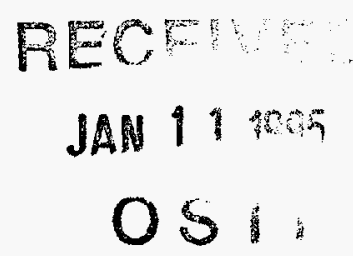

and

Peter von Ballmoos, Juan Naya, Francis Albernhe, G. Vedrenne

Centre d'Etude Spatial des Rayonnements, 9, av. du Colonel-Roche, 31029 Toulouse, France

and

Mohammed Faiz

King Fahd University of Petroleum and Minerals, Dhahran 31261, Saudi Arabia

October 1995

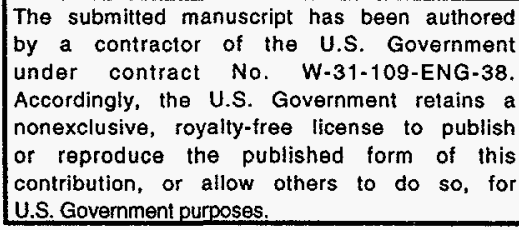

\section{DISCLAIMER}

\begin{abstract}
This report was prepared as an account of work sponsored by an agency of the United States Government. Neither the United States Government nor any agency thereof, nor any of their employees, makes any warranty, express or implied, or assumes any legal liability or responsibility for the accuracy, completeness, or usefulness of any information, apparatus, product, or process disclosed, or represents that its use would not infringe privately owned rights. Reference herein to any specific commercial product, process, or service by trade name, trademark, manufacturer, or otherwise does not necessarily constitute or imply its endorsement, recommendation, or favoring by the United States Government or any agency thereof. The views and opinions of authors expressed herein do not necessarily state or reflect those of the United States Government or any agency thereof.
\end{abstract}

Invited paper presented at the.Workshop on Imaging in High Energy Astronomy, Anacapri, Italy, September 26-30, 1995; to be published in proceedings book Imaging in High Energy Astronomy.

\footnotetext{
*This work supported by the U.S. Department of Energy, Basic Energy Sciences-Materials Sciences, under contract \#W-31-109-ENG-38.

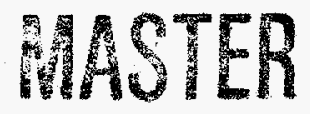




\section{Review of Crystal Diffraction and its Application to Focusing Energetic Gamma Rays}

\section{Robert K. Smither, Patricia B. Fernandez, Timothy Graber \\ Advanced Photon Source, Argonne National Laboratory,}

9700 S. Cass Avenue, Argonne, IL 60439 USA

and

Peter von Ballmoos, Juan Naya, Francis Albernhe, G. Vedrenne

Centre d Etude Spatiale des Rayonnements, 9, du Colonel-Roche, 31029 Toulouse, FRANCE

and

Mohammed Faiz

King Fahd University of Petroleum and Minerals, Dhahran 31261, SAUDI ARABIA

Invited paper presented at the Workshop on Imaging in High Energy Astronomy, Anacapri, Italy, Sept. 26-30, 1995. 


\title{
Review of Crystal Diffraction and its Application to Focusing Energetic Gamma Rays
}

\author{
Robert K. Smither, Patricia B. Fernandez, Timothy Graber \\ Advanced Photon Source, Argonne National Laboratory, \\ 9700 S. Cass Avenue, Argonne, IL 60439 USA
}

and

Peter yon Balimoos, Juan Naya, Francis Albernhe, G. Vedrenne

Centro d Etude Spatiale des Rayomements, 9, du Colonel-fioche, 31028 Toulouse, FRANCE

and

Mohammed Faiz

King Fahd University of Petroleum and Minerals, Dhahran 31261, SAUDI ARABIA

Abstract The basic features of crystal diffraction and their application to the construction of a crystal diffraction lens for focusing energetic gamma rays are described using examples from the work preformed at the Argonne National Laboratory. Both on-axis and off-axis performance are discussed. The review includes the use of normal crystals, bent crystals, and crystals with variable crystal-plane spacings to develop both condenser-type lenses and point-to-point imaging lenses.

\section{Introduction}

The basic design for a crystal tens that focuses high-energy gamma rays ${ }^{1-3}$ is shown in figure 1. One uses crystal diffraction from rings of crystals to collect gamma rays incident on a large area and concentrates them in a small focal spot for detection with a relatively small detector. Each ring uses a different set of crystalline planes

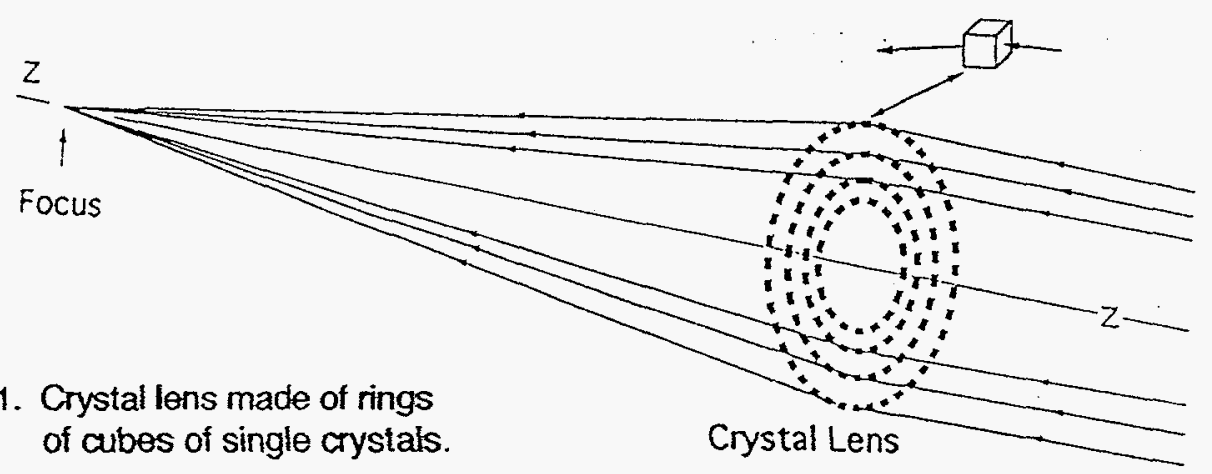

and/or a different crystalline material. The radius of each ring is adjusted so that the Bragg angle, $\theta$, is given by $\sin \theta=\lambda / 2 d$, where $\lambda$ is the wavelength of the gamma ray and $d$ is the spacing between crystalline planes. This approach can give one a large increase of signal in the detector without increasing the background in the detector and make a major improvement in the sensitivity of the instrument. Crystal-diffraction lenses divide into two basic types, Bragg lenses (surface-diffraction) and Laue lenses (volume-diffraction), which are commonly used for lower-energy gamma rays and high-energy gamma rays, respectively. Figure 2 compares these two cases. For energetic gamma rays the diffraction angle (Bragg angle) is quite small and Laue geometry makes much more efficient use of the crystalline material. Crystal lenses are further subdivided into condenser-type lenses that concentrate a large amount of radiation on a small focal spot 
and whose spatial resolution in the image plane is about the size of the individual diffraction crystals, ${ }^{1-6}$ and the point-to- point focusing type lenses $1,2,3,5,7,8$ that

mence chrstax.

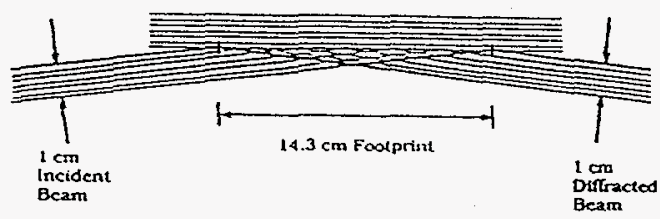

LAUE CRYSTAL

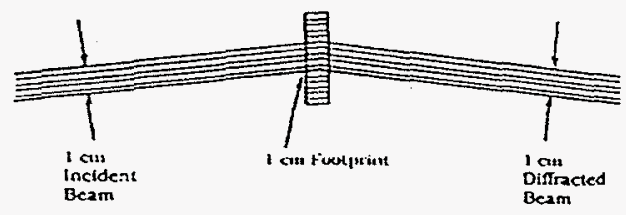

Figure 2. Comparison of Bragg and Laue Diffraction Crystals

produce real images of the source as well as a high concentration of photons at the focal point. These focusing point-to-point lenses come in two varieties. They both use crystals that are bent on a radius centered on the axis of the lens. One uses normal crystals and gives a focal spot with a diameter equal to the radial width of the crystal element. The other uses a bent variable metric crystal and has the potential of producing a focal spot of $0.1 \mathrm{~mm}$ or less for a point source and could generate an image with submillimeter spatial resolution. Argonne has been the leader in developing the Laue type lens for high-energy gamma rays and has experimented with all three different types mentioned above. Figure 3. compares the individual crystal lens elements for the unbent and bent cases.
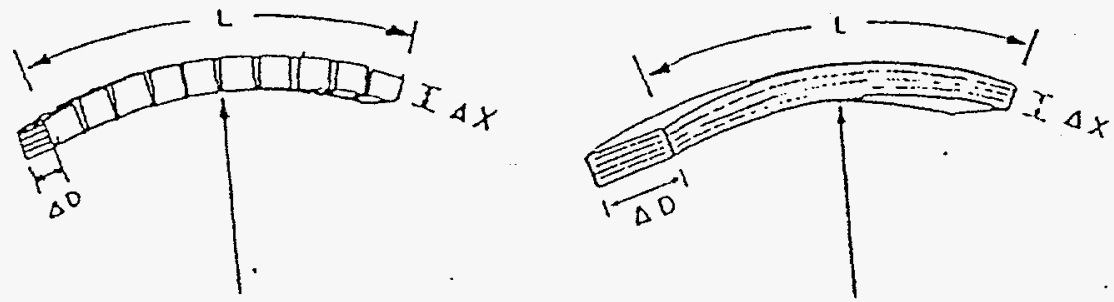

Figure 3. Comparison of the unbent and bent crystal lens elements.

Bending the crystal eliminates the spreading of the diffracted beam in the plane perpendicular to the diffraction plane (sagittal focusing). To focus the diffracted beam in the diffraction plane one must change the lattice spacing between the crystal planes as a function of radius. The spacing needs to decrease with increasing radius so that the Bragg angle can increase with radius and focus radiation diffracted from the whole crystal to a point focus. These bent crystal geometries are complicated and required large lens structures ( $1 \mathrm{~m}$ dia.) so only partial lens sections were built to demonstrate the principals involved.

\section{The Argonne Ge Crystal Lens}

The first complete lens constructed at Argonne was of the condenser type that used small cubes of normal (unbent) germanium crystals. The lens has a $45 \mathrm{~cm}$ diameter 
with 600 germanium crystals arranged in 8 rings. Figure 4 shows schematic drawing of

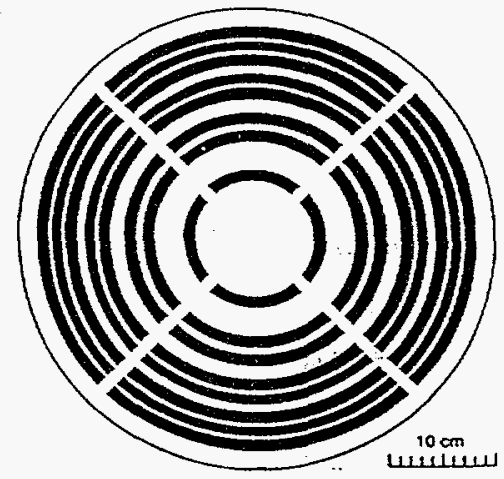

Figure 4. Schematic drawing of the crystal lens with outer dia of $90 \mathrm{~cm}$

this lens with its 8 rings of crystal cubes. Each crystal cube was mounted on an aluminum plate, one end of which was bolted to the lens frame and the other end was free to move. The diffraction angle of each crystal was adjusted separately by applying a light force on the free end of the aluminum plate through a soft spring. This resulted in a slight bending of the plate and allowed one to adjust each crystal so that the appropriate crystalline planes make the right Bragg angle $(+1-5$ arc sec) with the incident radiation to satisfy the required relation, $\lambda=2 \mathrm{~d} \sin \theta$. A $137 \mathrm{Cs}$ source of $661.65 \mathrm{keV}$ was used to align the the individual crystals, one at a time. Seven different gamma-ray energies have been used in the tests with this lens system. They are listed in Table I, with their source, full lens focal lengths, and distances from source to lens and lens to detector. All of these measurement were made without retuning the lens. All that was needed to focus a new energy was to change the distance from the source to the lens and place the detector at the new focal point as explained above.

\begin{tabular}{lcccc}
\hline $\begin{array}{l}\text { Energy } \\
\text { (keV) }\end{array}$ & Source & $\begin{array}{c}\text { Focal Length } \\
\text { (moters) }\end{array}$ & $\begin{array}{c}\text { Source-Lens } \\
\text { (meters) }\end{array}$ & $\begin{array}{c}\text { Lens-detector } \\
\text { (meters) }\end{array}$ \\
\hline 661.65 & $137 \mathrm{Cs}$ & 10.92 & 24.75 & 19.54 \\
511.00 & $22 \mathrm{Na}$ & 8.43 & 19.11 & 15.09 \\
413.7 & $239 \mathrm{Pu}$ & 6.83 & 15.47 & 12.22 \\
383.71 & $133 \mathrm{Ba}$ & 6.33 & 14.35 & 11.33 \\
375.2 & $239 \mathrm{Pu}$ & 6.19 & 14.03 & 11.08 \\
355.94 & $133 \mathrm{Ba}$ & 5.87 & 13.31 & 10.51 \\
302.83 & $133 \mathrm{Ba}$ & 5.00 & 11.33 & 9.70
\end{tabular}

Table 1: Gamma-ray sources tested with ANL germanium crystal lens.

\section{CRYSTAL DIFFRACTION FORMULA}

The crystal diffraction angle $\theta_{\mathrm{B}}$ is defined by equations:

$$
\sin \theta_{B}=n \lambda / 2 d
$$

and

$$
\lambda(A)=12.397 / E_{\gamma}(\mathrm{keV})
$$

where $\lambda$ is the wavelength of the garnma-ray (in Angstrom units in eq. 2), $d$ is the crystalline spacing, $n$ is he order of the diffraction, and $E_{\gamma}$ is the energy of the gamma-ray, in $\mathrm{keV}$. For a $100 \mathrm{keV}$ gamma-ray, diffracted be the [111] planes of germanium, $\theta_{\mathrm{B}}$ is about 1.0895 degrees, 0.01902 radians. Thus the surface of a Bragg diffraction crystal 
will need to be 52.6 times longer than the height of a Laue crystal used to diffract the same size beam. (see figure 2)

Each ring uses a different set of crystalline planes and can be of different crystalline material as well. The focal length (F.L.) for each ring (the distance from the ring to the focus for a distant source) is given by equation 3 ,

$$
\text { F.L. }=R / \tan 2 \theta
$$

where $R$ is the radius of the ring and $\theta$ is the Bragg diffraction angle defined above. By adjusting $R$ one can make the focal lengths of all the rings the same and obtain a single focus for the full lens. Substituting equations 1 \& 2 in 3

For small Bragg angles

$$
\text { F.L. }=R / \tan (2 \operatorname{arc}[(12.397) n / 2 d \text { Ey }])
$$

so for any ring with a given $R$ and $d$,

$$
F . L .=[R\{((12.397) \times n d(A)] \text { Ey }(\mathrm{keV})
$$

$$
F . L=\text { constant } \times \text { EY }
$$

If all of the rings have the same focal length, then eq. 6 is true for the full lens.

For a source at a finite distance from the lens, eq. 7 and 8 must be satisfied along with eq. $1 \& 2$.

$$
\begin{aligned}
& L_{S}=R / \tan (\theta-\alpha) \\
& L_{D}=R / \tan (\theta+\alpha)
\end{aligned}
$$

where $L_{S}$ is the distance from the source to the lens, $L_{D}$ is the distance from the lens to the detector, and $a$ is the angle between the crystalline planes and the axis of the lens. For the case of a distant source $a=q$. The small angle approach gives:

$$
F \cdot L=1 / F_{S}+1 / L_{D}
$$

which is the simple lens formula for a simple convex lens with visible light.

A typical scan over a small source (137 Cs) is shown in Figure 3. The background count rate is very small, less than 0.1 counts per sec., so the wings are a real part of the scan. These wings come about because the view of a single crystal is not a small circular (angular) area of space but rather a strip across the sky. This strip is narrow in the diffraction plane but can be quite wide in the plane perpendicular to the diffraction plane. This wide angular width depends on, the width of the individual crystals, the width of the

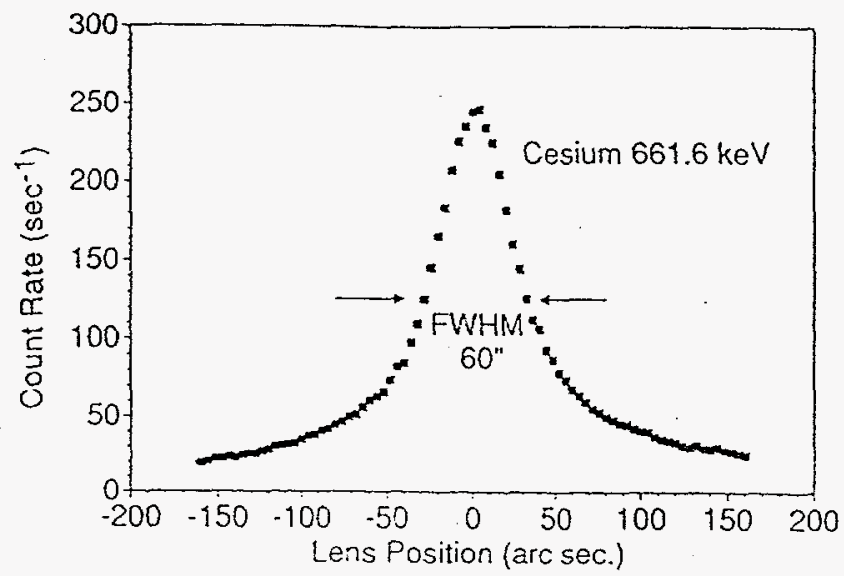

Figure 5. Vertical scan of crystal lens over a $3 \mathrm{~mm}$ dia. 137 Cs source.

detector and the distance from the lens to the detector. If the crystal is $2-\mathrm{cm}$ wide; the detector $6-\mathrm{cm}$ wide; and the distance from lens to detector is $800 \mathrm{~cm}$, then the angular width of the strip of the sky viewed by the crystal is 0.01 radians, which is 2063 arc seconds or 34.37 arc min. The wide wings seen in figure 5 would extend out either side of the peak by 1032 arc sec. 


\section{Off-Axis Response}

The wide wings in the field of view give the lens considerable off-axis sensitivity and make it much easier to locate a source. They also contribute significantly to the sensitivity (count rate) of the lens when the source has a finite angular size. For an extended source the wings can contribute more to the count rate in the detector than the central region of the field of view. This is because, atthough the sensitivity of the lens to off-axis sources decreases with the angular distance off-axis, the area being viewed increases at about the same rate. Thus each angular ring of the source contributes the same amount of focused flux to the diffracted beam. If the source exhibits a continuum energy spectrum, then a more complicated picture emerges. The corresponding view for an individual crystal for wavelengths other than that for which the lens focus was adjusted, will still be a strip of the sky but now it will be displaced from the center of the field of view. The strength of this response is strongest from a ring surrounding the center of the field of view. If the source is an extended source, then this wavelength will be focused on the detector as a ring surrounding the center of the detector and a series of wavelengths will be focused on the detector as a series of rings. If the source has an irregular shape, this shape will be imaged on the detector. If a monochromatic source is off center to the left, its image at the focal point will be off center to the right. Thus, the crystal lens has many of the features of a simple convex lens for visible light. This discussion suggests that a multi-element detector would be quite useful at the focal point of the lens, both for locating the source and for determining its the size and shape. Just such an experiment was performed at Argonne using a multi-element $\mathrm{Ge}$ detector supplied by the astrophysics group from Toulouse. This collaborative (Argonne + Toulouse) experiment is described in an other paper presented at this meeting.

\section{Angular Resolution and Diffraction Efficiency}

The angular resolution and the diffraction efficiency both depend on the physical properties of the crystals, alignment errors of the crystals, and on the design of the lens. These components are strongly coupled. Lenses that have good angular resolution tend to have good energy resolution, high diffraction efficiencies, large effective areas and low backgrounds for a narrow band width of gamma-ray energies. While lenses that are designed to focus large energy bandwidths tend to have poor angular resolution, smalter effective areas, and lower diffraction efficiencies for gamma-ray sources with narrow bandwidths. The full Argonne lens contains 600 germanium crystals in the form of $1 \mathrm{~cm}$ cubes, mounted in 8 rings. The diffraction planes used and the number of crystals in each ring are, respectively: [111], 28; [220], 52; [311], 60; [400], 76; [331], 84; [422], 92; [333], 100; [440], 108. They are all used in Laue (transmission) diffraction and have mosaic structures that are much larger than their Darwin widths. For a simple unbent crystal cube, the diffraction efficiency (the number of gamma rays focused on the detector divided by the number of gamma rays incident on the crystal) is the product of the transmission of the gamma rays through the crystal times the diffraction coefficient for the crystalline planes and is given by equation 11 ,

$$
\text { Diff. Eff. }=(\exp -\alpha x)[0.5(1-\exp \sigma x)]
$$

where $\alpha$ is linear absorption coefficient; $\sigma$ is the linear diffraction coefficient; and $x$ is the thickness of the Laue crystal. For the 661.65-keV line from $137 \mathrm{Cs}$, the transmission is $70 \%$ for a $1 \mathrm{~cm}$ germanium crystal and the maximum diffraction coefficient is $50 \%$, giving $35 \%$ for the maximum efficiency. The individual crystals have diffraction efficiencies for 
the Cs line that range from $4 \%$ to $15 \%$, depending on their mosaic structure and the crystalline planes used. These low efficiencies result from the use of crystals that are too perfect. The average acceptance angle of these crystals is 2 arc sec. The 3-mm dia. Cs source intercepts an angle of 25 arc sec as seen from the lens and the 1-cm crystats intercept an angie of 82 arc sec as seen from the source. Both of these subtended angles are much larger than the acceptance angle of the crystals so only a small region of the crystal can diffract gamma rays from a point in the source, and only a fraction of the crystal can diffract gamma rays from any part of the source. If these geometric effects are combined with the equation 11, they would predict that the diffraction efficiency of the lens would be less than 1 percent and its performance, very poor. The increase in the efficiency was obtained by cutting slots in the back of each crystal and wedging the three sectors apart so they made a $27-a r c-s e c$ angle with their adjacent sections. This reduced the miss match between the acceptance angles and the size of the crystals and the size of the source. Further improvements in the efficiency of the crystals was made by roughing the surfaces of the crystal. This increased the mosaic structure of small regions of the crystals near the surfaces. The net effect was to improve the efficiency from less than 1 percent to the 4 to 15 percent mentioned above.

Table II gives the diffraction efficiencies with the present lens for the measured sources, normalized to a constant distance from the source to the lens of $24.75 \mathrm{~m}$ and a constant diameter source, 3-mm dia., (25 arc sec). The last column gives the effective area of the full lens at that energy.

\begin{tabular}{cccc}
\hline Energy (keV) & Element & Diffraction Efficiency & Eff. Area, cm² \\
661.65 & $137 \mathrm{Cs}$ & 0.070 & 39. \\
551.00 & $22 \mathrm{Na}$ & 0.14 & 78. \\
413.7 & $239 \mathrm{Pu}$ & 0.22 & 123. \\
383.7 & $133 \mathrm{Ba}$ & 0.25 & 140. \\
355.9 & $133 \mathrm{Ba}$ & 0.27 & 151. \\
302.8 & $133 \mathrm{Ba}$ & 0.24 & 134. \\
\hline
\end{tabular}

Table 2: Diffraction efficiencies and effective areas for the $45 \mathrm{~cm}$ Lens

If the present crystals are replaced with crystals that have the optimum mosaic structure widths and the optimum thickness, the projected diffraction efficiency will be 25 to $40 \%$ for these gamma-ray energies. This corresponds to an effective area of 150 to $240 \mathrm{~cm}^{2}$. A lens with an effective area of this size should be quite acceptable for a balloon experiment. In all of these experiments, the direct beam from the source to the detector is blocked. If the central region of the lens is opened up so that the detector can see the source, then the effective areas will increase by the area of the detector $\left(28 \mathrm{~cm}^{2}\right)$.

The width of the scan over the Cs source (60 arc sec), as shown in Figure 3, is due to the width of the source ( 25 arc sec), the size of the 3 subsections (25 arc sec) of each crystal and the 1.6 size increase in width that comes from the circular geometry of the lens. The calculated width, 57 arc sec, is close to the measured value of 60 arc sec. Relatively littie of the width is due to the misalignment of the crystals.

\section{Standard and Multi-Element Detectors}

The Argonne lens system was tested with a standard intrinsic-germanium gammaray detector and also with a $3 \times 3$ matrix of germanium detectors brought to Argonne by 
the astrophysics group from Toulouse. ${ }^{8}$ More detail on the test results of this lens / matrixdetector combination can be found in the paper presented by Juan Naya at this conference.

\section{Tunable Crystal Lens}

The major advantage of the Argonne-type lens that adjusts all of the crystals to focus a narrow band of wavelengths and thus maximize its sensitivity for that wavelength, is also a major drawback for some experiments. Concentrating on one gamma-ray energy is all right for a balloon experiment, where the observation time is limited but it would not be acceptable for a satellite experiment, where one would like to view many different wavelengths. This means that one must be able to retune the lens to a new wavelength. The Toulouse - Argonne collaboration has devised at least two different ways of accomplishing this. Details on how this can be accomplished can found in a paper presented at this conference by Peter von Ballmoos. ${ }^{10}$

\section{Acknowledgement}

This work was supported in part by the DOE contract No. W31-109-38/ENG. The germanium crystal lens was designed and built with funds supplied by the DOE Office of Nonproliferation and National Security for the work preformed at ANL for the project entitled "Crystal Diffraction Lens For Long-Range Passive Detection Of Fissile Material".

\section{References:}

1. "New Method for Focusing X-Rays and Gamma-Rays", R.K. Smither, Rev. Sci. Instrum., 44, 131-141 (Feb. 1982).

2. "New Method for Focusing and Imaging $X$-Rays and Gamma-Rays with Diffraction Crystals", R. K. Smither, Sym. on Future X-Ray Experiments, X-Rays in the 80's", GSFC, Oct. 1981, NASA Tech. Mem. No. 83848 (Nov. 1981)

3. "A Positron Annihilation Radiation Telescope Using Laue Diffraction in a Crystal Lens", R. K. Smither and Peter von Ballmoos, INTEGRAL Workshop, Feb. 2-5 at Les Diablerets, Switzerland. AP supp., Vol. 92, 1994 June, 663

4. "A Bragg Crystal Fux Concentrator for Annihilation Radiation", R.K. Smither and N. Lund, 16th Inter. Cosmic Ray Conference, India (1983). Conference proceedings, supplement issue of AP.

5. "Crystal Diffraction Telescope for Discrete line sources", R.K. Smither, et. al., GRO Science Workshop, GSFC, April 1989, NASA Report, Ed, W. Neil Johnson.

6. "A Study of Focusing Telescopes for Soft Gamma Rays", Niels Lund, Experimental Astronomy Vol 2, (1992) 259

7. "Gamma-Ray Telescopes Using Variable-Metric Diffraction Crystals", R.K. Smither, 11th Texas Symposium on Relativistic Astrophysics", Austin, Texas, Dec. 1981, Annal. of New York Acad. Sci. 422 (1983) 384

8. "Crystal Diffraction Lenses for Imaging Gamma-Ray Telescope", R.K. Smither, "13th Texas Symposium on Relativistic Astrophysics," p 55-59, Ed. M.P. Ulmer, Northwestern Un., World Scientific Publishing Co., Singapore

9. "Experimental Results Obtained with the Positron-AnnihilationRadiation Telescope of the Toulouse-Argonne Collaboration", Juan Naya, et. al., contributed paper No. of this conference, $p$

10. "A Space Borne Crystal Diffraction Telescope for the Energy range of Nuclear Transitions", Peter von Ballmoos, et. al., contributed paper No. " " of this conference. 